\title{
The role of the Rayleigh-Taylor instability in ram pressure stripped disk galaxies
}

\author{
E. Roediger ${ }^{1,2}$ and G. Hensler ${ }^{3}$
}

\author{
1 Jacobs University Bremen, PO Box 750 561, 28725 Bremen, Germany \\ e-mail: e.roediger@jacobs-university.de \\ 2 Institute of Theoretical Physics and Astrophysics, University of Kiel, Olshausenstr. 40, 24098 Kiel, Germany \\ 3 Institute of Astronomy, University of Vienna, Türkenschanzstrasse 17, 1180 Vienna, Austria \\ e-mail: hensler@astro.univie.ac.at
}

Received 22 January 2008 / Accepted 5 February 2008

\section{ABSTRACT}

\begin{abstract}
Ram pressure stripping, i.e. the removal of a galaxy's gas disk due to its motion through the intracluster medium of a galaxy cluster, appears to be a common phenomenon. Not every galaxy, however, is completely stripped of its gas disk. If the ram pressure is insufficiently strong, only the outer parts of the gas disk are removed, and the inner gas disk is retained by the galaxy. One example of such a case is the Virgo spiral NGC 4402. Observations of NGC 4402 (Crowl et al. 2005, AJ, 130, 65) reveal structures at the leading edge of the gas disk, which resemble the characteristic finger-like structures produced by the Rayleigh-Taylor (RT) instability. We argue, however, that the RT instability is unlikely to be responsible for these structures. We demonstrate that the conditions under which a galaxy's disk gas experiences ram pressure stripping are identical to those that lead to RT instability. If the galaxy's gravity prevents ram pressure stripping of the inner disk, it also prevents the RT instability. In contrast, the stripped gas could still be subject to RT instability, and we discuss consequences for the stripped gas.
\end{abstract}

Key words. galaxies: spiral - galaxies: evolution - galaxies: ISM - galaxies: intergalactic medium - instabilities

\section{Introduction}

Differences between field and cluster galaxies imply that the evolution of galaxies is influenced strongly by their environments. In addition to the gravitational interaction between the cluster galaxies themselves, ram pressure stripping (RPS), the (partial) removal of a galaxy's gas due to its motion through the intracluster medium (ICM), is thought to play an important role for the evolution of galaxies (see e.g. van Gorkom 2004).

RPS has been modelled numerically by different groups (e.g. Abadi et al. 1999; Schulz \& Struck 2001; Quilis et al. 2000; Vollmer et al. 2001; Marcolini et al. 2003; Roediger \& Hensler 2005, hereafter RH05; Roediger \& Brüggen 2006, hereafter RB06; Roediger et al. 2006; Roediger \& Brüggen 2007, hereafter RB07). In environments in which ram pressure stripping is prevalent, such as cluster centres, the stripping is very effective, but the central part of the gas disk can be retained by the galaxy for quite some time.

In principle, the remaining gas disk can be affected by the Rayleigh-Taylor (RT) instability, which occurs when a lighter fluid supports a heavier one against a gravitational field or an acceleration. An equivalent situation occurs when a denser fluid is accelerated by a lighter one, e.g. when a dense gas cloud is exposed to a wind of rarefied gas (or when a gas cloud moves through this ambient gas). Such a gas cloud is expected to be destroyed by the RT instability, and subsequent KelvinHelmholtz $(\mathrm{KH})$ instability, unless stabilising mechanisms are in place such as (self-)gravity, surface tension, magnetic fields (Chandrasekhar 1961), or heat conduction (Vieser \& Hensler 2007).
Thus, as a (disk) galaxy moves through the ICM and its gas is stripped by RPS, it should be affected by the RT instability. The detection of a filamentary leading surface in NGC 4402, a Virgo cluster galaxy with clear signs of RPS, lends support to the idea that the RT instability does affect the remaining gas disk. Recent images by Crowl et al. (2005) reveal finger-like structures at the galaxy's upstream side, which are slightly inclined with respect to the rotation axis of the disk. If these pillars are interpreted as the results of the RT instability, the direction of motion of the galaxy that they imply would be identical to that implied by HI observations (Crowl et al. 2005).

Such structures are not, however, seen in the simulations. In Sect. 3.1, we demonstrate analytically that the RT instability is suppressed in the remaining gas disk due to the gravitation of the galaxy. In addition, we discuss the role of the RT instability in deciding the fate of stripped gas in Sect. 3.2. To start with, we summarise basic analytical estimates concerning the RT instability in Sect. 2.

\section{The Rayleigh-Taylor instability}

A detailed derivation of stability conditions and instability growth rates is provided by Chandrasekhar (1961).

We consider the simplest case of two superimposed, incompressible, inviscid, unmagnetised fluids. These fluids are separated by a contact discontinuity that is perpendicular to the effective homogeneous (gravitational) acceleration, $a$. Referring to the direction of $a$ as "downwards", this setup is unstable if the upper fluid has a higher density than the lower one. Any perturbation at the interface will be amplified by the RT instability, developing characteristic finger-like structures. In the linear 


\section{rest frame of ambient medium}

\section{cloud's rest frame}
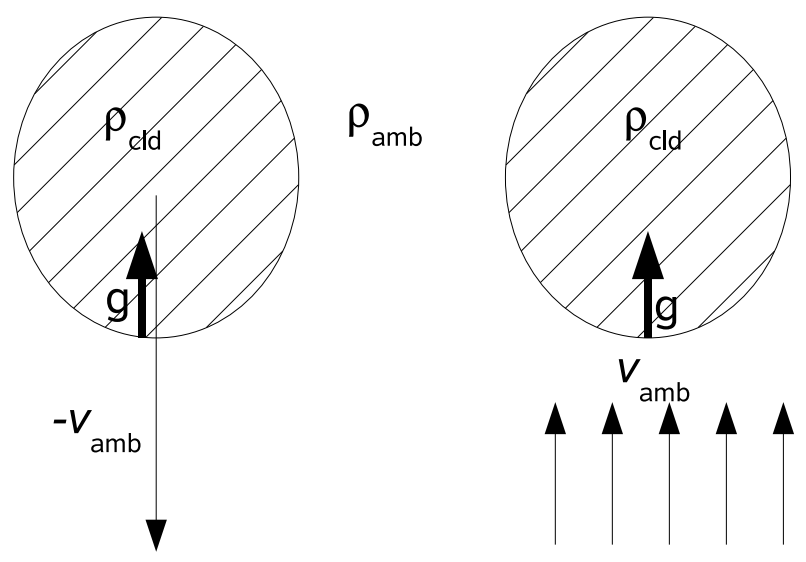

Fig. 1. Sketch of a cloud moving through an ambient medium, in the rest frame of the ambient medium (left), and in the cloud's rest frame (right). The direction of the gravitational acceleration at the cloud's upstream side, due to its self-gravity, is shown by the vector $g$.

regime, small perturbations grow at the most rapid rate. Also a continuous stratification will be unstable if the density increases upwards.

Here we are interested in the situation sketched in Fig. 1: a dense cloud of mass, $M_{\mathrm{cl}}$, density, $\rho_{\mathrm{cl}}$, and cross section $A$ is moving with velocity, $-v_{\mathrm{amb}}$, through an ambient gas of density $\rho_{\mathrm{amb}}$, or, equivalently, is exposed to a wind of ambient gas with velocity $v_{\mathrm{amb}}$. The relevant acceleration is the one exerted by the drag force $F_{\mathrm{D}}$ :

$$
\begin{aligned}
a_{\mathrm{D}} & =\frac{F_{\mathrm{D}}}{M_{\mathrm{cl}}}=\frac{\frac{1}{2} c_{W} A \rho_{\mathrm{amb}} v_{\mathrm{amb}}^{2}}{M_{\mathrm{cl}}} \\
& =\frac{\frac{1}{2} c_{W} \rho_{\mathrm{amb}} v_{\mathrm{amb}}^{2}}{\Sigma_{\mathrm{cl}}}=\frac{1}{2} c_{W} \frac{p_{\mathrm{ram}}}{\Sigma_{\mathrm{cl}}},
\end{aligned}
$$

where $c_{W}$ is the drag coefficient, and the mean column density of the cloud is given by

$\Sigma_{\mathrm{cl}}=\frac{M_{\mathrm{cl}}}{A}=\frac{4 / 3 \pi R_{\mathrm{cl}}^{3} \rho_{\mathrm{cl}}}{\pi R_{\mathrm{cl}}^{2}}=4 / 3 R_{\mathrm{cl}} \rho_{\mathrm{cl}}$.

The drag coefficient depends on the cloud's shape and surface properties, where $\frac{1}{2} c_{W} \sim 1$ may be used as an approximation. At the upstream side of the cloud, in the rest frame of the cloud the drag force appears as a pseudo force pointing upstream, the correct direction to produce the RT instability at the cloud's upstream edge.

A possible way to stabilise the cloud's surface against the RT instability would be a gravitational field associated with the cloud. The gravitational acceleration, $g$, at the cloud's upstream edge works in the opposite direction of $a_{\mathrm{D}}$ ("inwards" for the cloud), so that the relevant acceleration for the development of the RT instability is the effective acceleration

$a_{\mathrm{eff}}=-a_{\mathrm{D}}+g$.

The RT instability will be suppressed, if $a_{\text {eff }}>0$, or

$g>a_{\mathrm{D}}$
Another mechanism to stabilise the cloud against RT instability, is provided by magnetic fields perpendicular to the cloud surface. Magnetic fields act like a surface tension and suppress the growth of perturbations of scale-length $\lambda<\lambda_{\mathrm{c}}$ with

$\lambda_{\mathrm{c}}=\frac{B^{2} \cos ^{2} \theta}{\mu_{0}\left(\rho_{\mathrm{cl}}-\rho_{\mathrm{amb}}\right) a_{\mathrm{D}}}=\frac{B^{2} \cos ^{2} \theta \Sigma_{\mathrm{cl}}}{\mu_{0} \rho_{\mathrm{cl}} \frac{1}{2} c_{W} \rho_{\mathrm{amb}} v_{\mathrm{amb}}^{2}}$,

where $\theta$ is the angle between the magnetic field direction and the direction of the perturbation (Chandrasekhar 1961). We assume that $\rho_{\mathrm{cl}} \gg \rho_{\mathrm{amb}}$. If $\lambda_{\mathrm{c}}$ is larger than the size of the cloud, it should therefore be resistant to RT instability because the largest possible perturbations have a size that is similar to that of the cloud.

\section{Application}

\subsection{The remaining gas disk}

We now investigate to what extent the RT instability can develop in a disk galaxy that is either moving face-on through the ICM, or is exposed to an ICM wind of density $\rho_{\text {ICM }}$ and velocity $v_{\text {ICM }}$. In Fig. 1, we replace the cloud by a disk galaxy with gas surface density, $\Sigma_{\text {gas }}$. The gravitational acceleration due to the galaxy potential, $g$, at the upstream side of the galaxy, points downstream. According to Eq. (4), the condition for RT instability to occur is

$g<\frac{1}{2} c_{\mathrm{W}} \frac{\rho_{\mathrm{ICM}} v_{\mathrm{ICM}}^{2}}{\Sigma_{\text {gas }}}$

or $\quad g \Sigma_{\text {gas }}<\frac{1}{2} c_{\mathrm{w}} \rho_{\mathrm{ICM}} v_{\mathrm{ICM}}^{2}$.

For a disk galaxy, both, $g$ and $\Sigma_{\text {gas }}$ depend on the radial position within the disk and both typically decrease with disk radius, $r$. In the outer parts (where $g \Sigma_{\text {gas }}$ is smaller), the RT instability can occur, while it is suppressed in the inner part, where $g \Sigma_{\text {gas }}$ is larger. An identical criterion is applied to decide the radius out to which gas will be stripped from the disk by ram-pressure (see e.g. RH05, RB06). The ram pressure, $\frac{1}{2} c_{\mathrm{w}} \rho_{\mathrm{ICM}} v_{\mathrm{ICM}}^{2}$, can be compared to the gravitational restoring force, $g \Sigma_{\text {gas }}$, as written in Eq. (7). At radii where this inequality applies, the gas will be ram pressure stripped; if the inequality does not apply, the expression on its left-hand side will dominate, and the galaxy retains its gas. In this second case, the inner gas disk is not stripped and is protected against RT instability by the gravity of the galaxy. This behaviour is reproduced by simulations (see e.g. Fig. 2).

We note that tangential magnetic fields would stabilise the remaining disk even further.

\subsection{The stripped gas}

According to the results of the previous section, the galaxy's gravitational field cannot prevent the RT instability of the stripped material. In the absence of other stabilising effects, the stripped gas should therefore fragmentate. The simulations of e.g. RH05, RB06 and RB07 neglected all possible stabilising effects, and therefore led to fragmentation. Figure 2 displays two simulation snapshots that differ only in spatial resolution. The resolution fixes the lengthscale of the smallest possible perturbations. According to analytical estimates, the smallest perturbations grow most rapidly, thus the stripped gas should fragmentate into smaller clouds, in simulations of higher resolution. This behaviour is in fact observed in, both, our 2D and 3D simulations. 

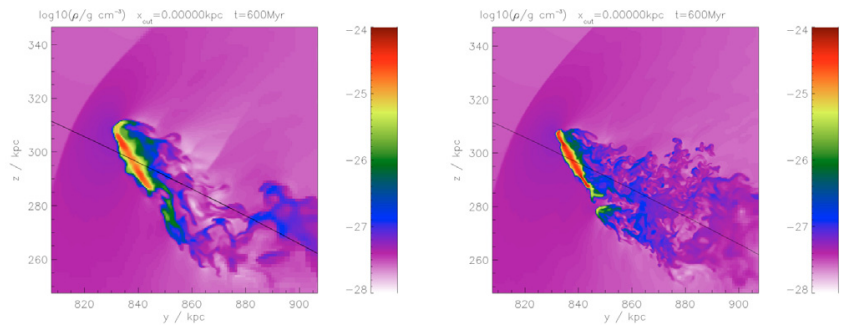

Fig. 2. Simulation snapshots from simulations described in RB07. Identical conditions except for the resolution: in rhs panel, the spatial resolution is 2 times better than in the lhs panel. The remaining gas disk is not fragmented, but the stripped gas is. The fragmentation is stronger for higher resolution.

\subsubsection{Self-gravity}

We consider the extent to which self-gravity of stripped gas clouds can prevent RT instability. This case will apply at the cloud's surface, if the gravitational acceleration exceeds $a_{\mathrm{D}}$ (Eq. (4)). If we assume a spherical gas cloud of density, $\rho_{\mathrm{cl}}$, and radius, $R_{\mathrm{cl}}$, after completing some simple arithmetic, we find that the RT instability is suppressed if

$$
\begin{aligned}
\frac{\Sigma_{\mathrm{cl}}}{3 \times 10^{21} \mathrm{~cm}^{-2}}= & \frac{n_{\mathrm{cl}}}{\mathrm{cm}^{-3}} \frac{R_{\mathrm{cl}}}{\mathrm{kpc}}>\frac{1}{2}\left(\frac{\rho_{\mathrm{ICM}}}{10^{-27} \mathrm{~g} \mathrm{~cm}^{-3}}\right)^{1 / 2} \\
& \times\left(\frac{v_{\mathrm{ICM}}}{1000 \mathrm{~km} \mathrm{~s}^{-1}}\right)\left(\frac{c_{W}}{0.5}\right)^{1 / 2} .
\end{aligned}
$$

This implies that only clouds of high column densities can prevent an RT instability. We remark, however, that these clouds may yet be affected and dissolved, by the KH instability.

\subsubsection{Magnetic fields}

A second mechanism to stabilise stripped-off clouds against RT instability are tangential magnetic fields. We assume again a spherical gas cloud of radius, $R_{\mathrm{cl}}$, and substitute the expression for $\Sigma_{\mathrm{cl}}$, from Eq. (2) into Eq. (5) to obtain

$$
\begin{aligned}
\lambda_{\mathrm{c}}= & \frac{B^{2} \cos ^{2} \theta \frac{4}{3} R_{\mathrm{cl}}}{\mu_{0} \frac{1}{2} c_{W} \rho_{\mathrm{ICM}} v_{\mathrm{ICM}}^{2}}=1.06 \cdot R_{\mathrm{cl}} \cos ^{2} \theta\left(\frac{B}{5 \mu G}\right)^{2} \\
& \times\left(\frac{\rho_{\mathrm{ICM}}}{10^{-27} \mathrm{~g} \mathrm{~cm}^{-3}}\right)^{-1}\left(\frac{v_{\mathrm{ICM}}}{1000 \mathrm{~km} \mathrm{~s}^{-1}}\right)^{-2}\left(\frac{c_{W}}{0.5}\right)^{-1} .
\end{aligned}
$$

This result is independent of the density of the cloud, and compares the energy density of the ICM wind with the energy density of the magnetic field. The magnetic field in spiral galaxies consists of an ordered large-scale field of approximately $1 \mu \mathrm{G}$, and a small-scale, tangled component with a field strength of about $5 \mu \mathrm{G}$ (see Lequeux 2005, and references therein). We therefore assume that because of the small-scale field, a magnetic field $B$-component tangential to the cloud's surface with field strength of the order of a few $\mu \mathrm{G}$ will exist. The critical value of $\lambda_{\mathrm{c}}$ is then of the same order as the radius of the cloud. It is therefore likely that the stripped gas will suffer RT instability only in case of strong ICM winds.

\subsubsection{Kelvin-Helmholtz-instability}

In addition to the RT instability, the stripped gas can suffer KH instability. This instability cannot be prevented by a gravitational field, but can be averted by tangential magnetic fields.
We note that the relevant surface for $\mathrm{KH}$ instability are the sides of the gas clouds. For tangled magnetic fields however, a tangential component should be found. The criterion to suppress the $\mathrm{KH}$ instability is similar to before. It is suppressed when

$$
\begin{aligned}
1< & \frac{B^{2}}{2 \pi \mu_{0} \rho_{\mathrm{ICM}} v_{\mathrm{ICM}}^{2}}=0.037\left(\frac{B}{5 \mu G}\right)^{2} \\
& \times\left(\frac{\rho_{\mathrm{ICM}}}{10^{-27} \mathrm{~g} \mathrm{~cm}^{-3}}\right)^{-1}\left(\frac{v_{\mathrm{ICM}}}{1000 \mathrm{~km} \mathrm{~s}^{-1}}\right)^{-2},
\end{aligned}
$$

implying that the $\mathrm{KH}$ instability occurs in weaker winds, even if the RT instability does not take effect, apart from very weak winds such as those in the cluster outskirts.

\section{Discussion}

In this paper, we have derived two main results. We have shown that the inner parts of ram pressure stripped disk galaxies, which are resistant to RPS, are in addition stable against RT instability in the case of face-on stripping. The same should be true at mild inclinations, as neither the gravitational acceleration in the wind direction, nor the projected gas surface-density changes significantly with inclination angle. In addition, the clumps of strippedoff gas are exposed to RT instability and should be destroyed.

Given that the RT instability is suppressed for the inner gas disk that survives ram pressure stripping, we conclude that the structures observed at the leading edge of NGC 4402 are not caused by the RT instability. These structures could be caused by different processes, one possibility being the inhomogeneous ISM as discussed by Crowl et al. (2005). Quilis et al. (2000) first studied the effect of an inhomogeneous ISM on ram-pressure stripping simulations, by creating holes inside the gas disk. The holes produced more pronounced Kelvin-Helmholtz instabilities, and thus stronger mass loss. The observations of NGC 4402 do not concern under-dense but over-dense regions in the ISM: dense gas clumps appear to be left behind after the diffuse gas is stripped, but the dense clouds also appear to be ablated. To follow such structures in simulations requires extremely high resolution and computational effort. Nonetheless, this is an important task for future simulations.

To what extent could stripped gas be observed? A complete investigation of this question would have to include processes such as thermal conduction and evaporation, cooling and star formation, as well as the effects of RT and KH instabilities. The above estimates imply that the stripped gas is likely affected by RT instability, and possibly also KH instability. Nevertheless, more massive stripped-off gas packages can be gravitationally bound and stable against RT instability. Qualitatively, this can explain why only a few massive clouds are visible (in $\mathrm{H}_{\alpha}$ ) for galaxies observed being affected by RPS (such as e.g. NGC 4522: Kenney \& Koopmann 1999: and NGC 4569: Fig. 4 in Tschoeke et al. 2001) while in smaller clumps the destructive RT instability dominates and disperses the gas into the ICM. The fact that the stable, stripped-off clouds emit $\mathrm{H}_{\alpha}$ in spite of no stars having formed, implies that they are heated by external sources. As the most plausible candidate, heat conduction should be suggested because, in contrast to general assumptions, self-gravitating clouds are not destroyed by evaporation but accumulate gas by condensation (Vieser \& Hensler 2007), and are, furthermore, stabilised against KH instability. Vollmer et al. (2001) discussed the influence of thermal conduction and effect of reduced heating on stripped gas clouds. They argued that the clouds no longer absorb the stellar far-ultraviolet radiation field. 
They conclude that the stripped gas could be either ionised and hot, or in the form of cool and even molecular clouds, enclosed in neutral atomic shells. More detailed studies are required to study the combined influence of these effects, as well as the influence of star formation.

Acknowledgements. This work was supported by the Deutsche Forschungsgemeinschaft project number He 1487/30. We gratefully acknowledge fruitful and helpful discussions with Marcus Brüggen, Joachim Köppen, Bernd Vollmer and Curtis Struck.

\section{References}

Abadi, M. G., Moore, B., \& Bower, R. G. 1999, MNRAS, 308, 947 Chandrasekhar, S. 1961, Hydrodynamic and Hydrostatic Stability (Oxford University Press)
Crowl, H. H., Kenney, J. D. P., van Gorkom, J. H., \& Vollmer, B. 2005, AJ, 130, 65

Kenney, J. D. P., \& Koopmann, R. A. 1999, AJ, 117, 181

Lequeux, J. 2005, The interstellar medium (Berlin, Heidelberg: Springer)

Marcolini, A., Brighenti, F., \& D'Ercole, A. 2003, MNRAS, 345, 1329

Quilis, V., Moore, B., \& Bower, R. 2000, Science, 288, 1617

Roediger, E., \& Brüggen, M. 2006, MNRAS, 369, 567

Roediger, E., \& Brüggen, M. 2007, MNRAS, 380, 1399

Roediger, E., \& Hensler, G. 2005, A\&A, 433, 875

Roediger, E., Brüggen, M., \& Hoeft, M. 2006, MNRAS, 371, 609

Schulz, S., \& Struck, C. 2001, MNRAS, 328, 185

Tschoeke, D., Bomans, D. J., Hensler, G., \& Junkes, N. 2001, A\&A, 380, 40

van Gorkom, J. H. 2004, in Carnegie Observatories Astrophysics Series, Clusters of Galaxies: Probes of Cosmological Structure and Galaxy Evolution, ed. J. S. Mulchaey, A. Dressler, \& A. Oemler (Cambridge: Cambridge University Press), 3, 306

Vieser, W., \& Hensler, G. 2007, A\&A, 472, 141

Vollmer, B., Cayatte, V., Balkowski, C., \& Duschl, W. J. 2001, ApJ, 561, 708 